\title{
Primary mesenchymal chondrosarcoma of the pancreas
}

\author{
XIANMIN BU, XIANWEI DAI \\ Department of Hepatopancreatobiliary Surgery, Shengjing Hospital, China Medical University, Shenyang, Liaoning Province, \\ China
}

\begin{abstract}
Extraskeletal chondrosarcomas are rare and there is only one reported case of primary pancreatic chondrosarcoma. We report the case of a 34-year-old woman with a 6-month history of abdominal pain and distention. Radiological studies indicated a mass in the pancreas, and exploratory laparotomy revealed a tumour of the pancreas extending to the hepatic vessels and hepatoduodenal ligament. The mass was completely excised, and the histopathological diagnosis was primary mesenchymal pancreatic chondrosarcoma. Tumour recurred at follow-up 52 months postoperatively.
\end{abstract}

Keywords: Chondrosarcoma - Pancreas - Pancreatic chondrosarcoma

Chondrosarcoma is a highly malignant, relatively common tumour of bone, but extraskeletal chondrosarcoma has only infrequently been reported in the medical literature. ${ }^{1}$ Pancreatic chondrosarcoma is extremely rare, with only a few reports in the literature, and most cases involve metastasis to pancreas from other locations. ${ }^{2}$ There is only one report in the literature of primary pancreatic chondrosarcoma. ${ }^{3}$ We herein report the case of a 34-year-old woman with primary pancreatic chondrosarcoma.

\section{Case history}

A 34-year-old woman was seen with complaints of a 6month history of abdominal distention, pain, and extreme discomfort in the upper abdomen after meals. The patient denied any significant past medical or past surgical history. An abdominal mass was found on examination and she was admitted for work-up. Physical examination revealed a solid, irregular, hard mass with unclear boundaries in the left upper quadrant. There was little mobility to the mass, and the patient experienced pain with palpation. The patient's physical examination was otherwise unremarkable. Laboratory studies revealed a bilirubin level of $19.2 \mathrm{mmol} / 1$ (normal, 0-20.5 mmol/1), albumin, $37.6 \mathrm{~g} / 1$ (normal, 35-55 g/1); aminotransferase, $25 \mathrm{U} / 1$ (normal, 0-40 U/1), carcinoembryonic antigen (CEA), $2.1 \mathrm{ng} / 1$ (normal, 0-5 ng/1); $\alpha$-fetoprotein (AFP), $3.3 \mathrm{ng} / 1$ (normal, 0-9 ng/1); cancer antigen (CA)19-9, 23 $\mathrm{U} / \mathrm{ml}$ (normal, 0-37 U/ml).

Computed tomographic (CT) scan of the abdomen demonstrated a large tumour mass of mixed density with calcifications in the pancreas (Fig. 1). The mass enveloped retroperitoneal blood vessels and protruded into the hepatoduodenal ligament. The primary stem of the portal vein was obstructed, and collateral circulation of the

Correspondence to: Xianwei Dai MD, Department of Hepatopancreatobiliary Surgery, Shengjing Hospital, China Medical University, No. 36 Sanhao Street Heping District, Shenyang, Liaoning Province, China I 10004

E: buxianmin@।63.com 


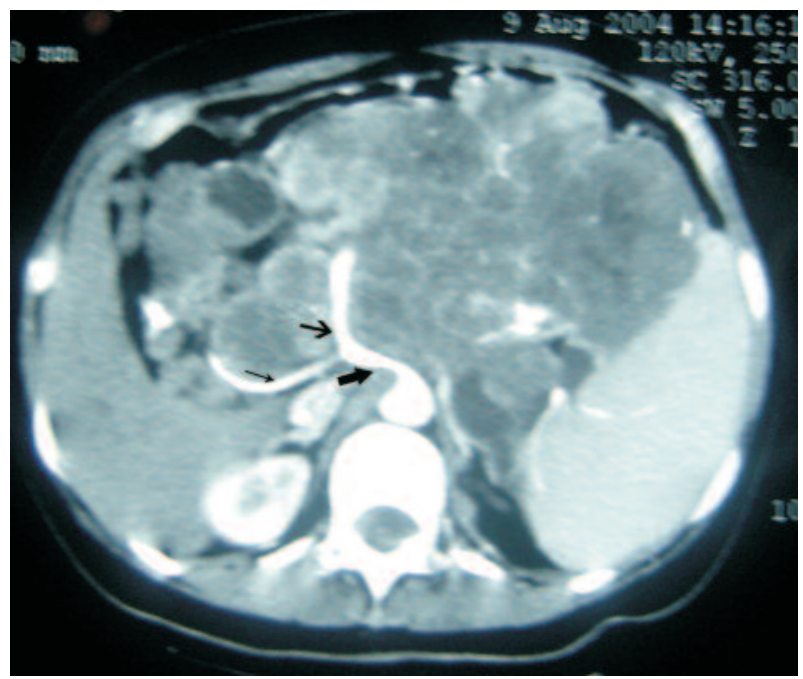

Figure I Computed tomographic examination indicates a large mass at the body and tail of the pancreas pressing against retroperitoneal blood vessels and extending into the hepatoduodenal ligament. Thin arrow, common hepatic artery; thick arrow, celiac trunk; medium arrow, splenic artery.

portal vein was evident. The splenic vein was displaced forward, and the celiac trunk and its branch and splenic artery were enveloped within the mass. Chest X-ray was normal and whole body single photon emission computed tomography (SPECT) scan revealed no evidence of metastasis.

An exploratory laparotomy was performed and the body and tail of the pancreas and the spleen were removed. Intra-operatively, only minimal normal pancreatic tissue was noted in the head of pancreas and the uncinate process. The body and tail of the pancreas was entirely occupied by the large mass, and measured approximately $18 \mathrm{~cm} \times 16 \mathrm{~cm}$. The spleen was enlarged, but otherwise appeared normal. Tumour protruded into hepatoduodenal ligament and was pressing on portal vein and hepatic artery, though no infiltration of the vascular wall was seen. No metastasis to the liver or other internal organs was noted. The surrounding lymph nodes were not enlarged. The mass was completely removed in two steps. First, the spleen and a large section of the mass were removed. Next, the abdominal aorta, celiac trunk, and superior mesenteric artery were exposed and protected. Then, the tumour in hepatoduodenal ligament was removed and the normal tissue at the head of pancreas was preserved.

The gross appearance of the mass was nodular; on cross-section, it was a greyish-white in colour and diffusely distributed cartilaginous tissue was seen. Microscopic examination revealed the tumour to be composed of undifferentiated mesenchymal cells (Fig. 2). The cells were round to spindle-shaped, homogeneous in

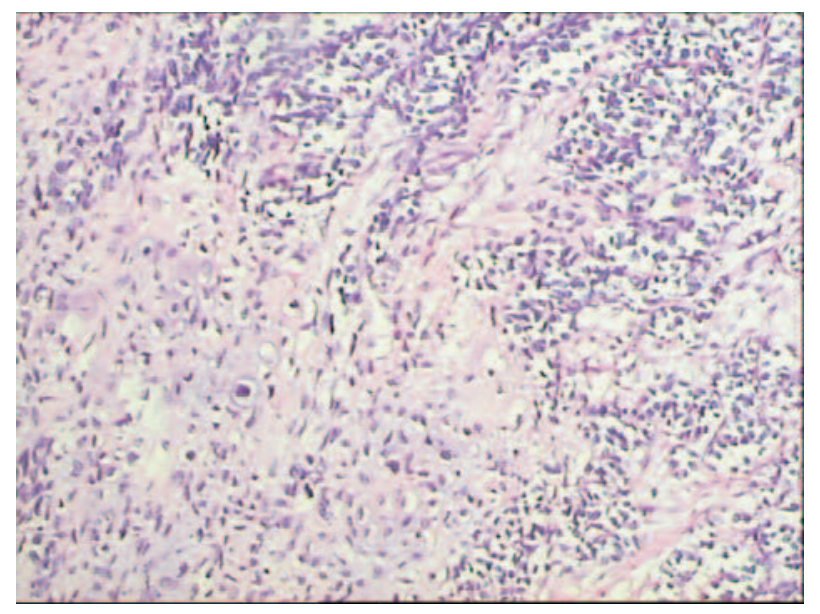

Figure 2 Histological examination reveals small round or spindleshaped mesenchymal cells and islets of highly differentiated cartilage (haematoxylin and eosin, $\times 100$ ).

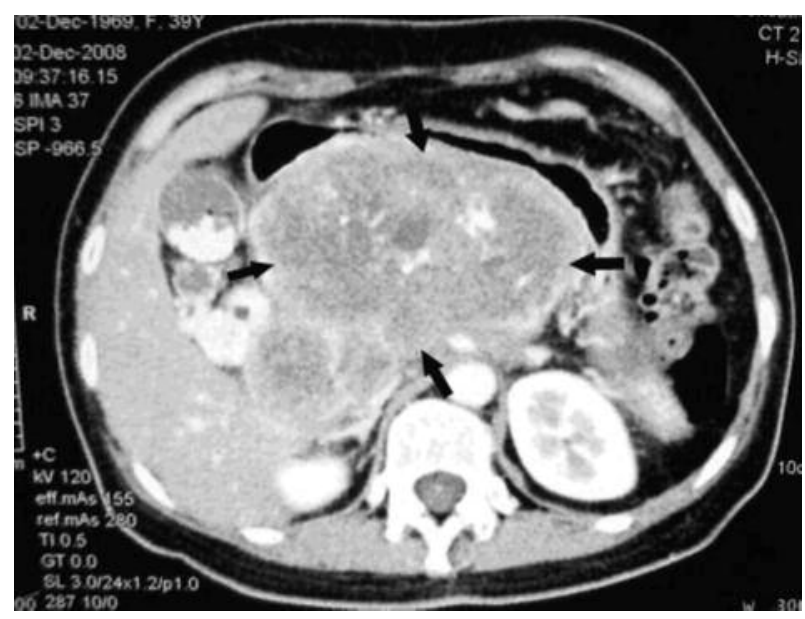

Figure 3 At 52 months postoperatively, computed tomography reveals in situ recurrence of the tumour. The arrows indicate the extent of the tumour. Peritoneal growth of the tumour can be seen.

size with scant cytoplasm, and heavily staining nuclei and less prominently staining cytoplasm. Unequal nuclear fission was noted. Islets of differentiated cartilage were observed. Examination of the edge of pancreas revealed no residue tumour. The final histopathological diagnosis was pancreatic mesenchymal chondrosarcoma.

The patient was discharged in good condition 17 days after surgery. Postoperative adjuvant chemotherapy and radiotherapy were recommended; however, the patient declined. The patient was followed-up every 3-6 months with CT scans. At follow-up 52 months after surgery, CT examination revealed the in situ recurrence of tumour (Fig. 3), but no remote metastases were observed. The patient declined further treatment and was lost to follow-up. 


\section{Discussion}

Extraskeletal chondrosarcoma refers to the presence of primary chondrosarcoma in soft tissues. The condition was first reported by Stout and Verner ${ }^{4}$ in 1953. Its incidence rate is far lower than osteochondrosarcoma; a report by the Rizzoli Center indicated that only 9 out of 513 cases of chondrosarcoma were extraskeletal. ${ }^{5}$ Extraskeletal chondrosarcoma is primarily seen in the head and neck in the ocular orbits, brain, and dural membrane. ${ }^{6}$ Chondrosarcoma occurs less frequently in other locations including the lower limbs, chest wall, and posterior peritoneal membrane, and has been rarely observed to occur in the uterus. ${ }^{7}$ Primary pancreatic chondrosarcoma is extremely rare; there has been only one other case reported in the literature. ${ }^{3}$

Histological classification of chondrosarcoma includes myxoid, mesenchymal, and dedifferentiated types. Mesenchymal chondrosarcoma was first reported by Lichtenstein and Bernstein ${ }^{8}$ in 1959, and extraskeletal mesenchymal chondrosarcoma in soft tissues was reported by Dowling ${ }^{9}$ in 1964 . The incidence of extraskeletal mesenchymal chondrosarcoma is very low; a report by Shapeero et al. ${ }^{7}$ showed that only 7 out of 224 cases of mesenchymal chondrosarcoma were actually extraskeletal in origin. The disease typically presents between the ages of 15-35 years, and there is a higher incidence in females than males. ${ }^{10}$

Chondrosarcoma is an aggressive malignancy, and primary therapy is surgical. Although adjuvant chemotherapy or radiotherapy may be of benefit, their therapeutic effects remain uncertain. ${ }^{1}$ Operative management depends on the location of tumour. In the case presented, it was essential to protect major blood vessels such as the celiac trunk, superior mesenteric artery, and portal vein. The absence of tumour invasion into the vascular of the involved vessels was of benefit in this case, as was the lack of lymph node involvement.

The prognosis for individuals with mesenchymal chondrosarcoma is poor because early recurrence and remote metastases occur frequently. ${ }^{1,6,7}$ Survival rates at 5 and 10 years are reported to be $54.6 \%$ and $27.3 \%$, respectively. ${ }^{10}$

\section{Conclusions}

Primary extraskeletal chondrosarcoma is a rare disease with high malignant potential and poor prognosis. Surgical excision is the primary mode of treatment, and the value of chemotherapy or radiotherapy has not been determined. In the case presented, the prognosis was better than if primary pancreatic cancer had been discovered.

\section{References}

1. Gelderblom H, Hogendoorn PC, Dijkstra SD, van Rijswijk CS, Krol $\mathrm{AD}$, Taminiau $\mathrm{AH}$ et al. The clinical approach towards chondrosarcoma. Oncologist 2008; 13: 320-9.

2. Chatzipantelis P, Karvouni E, Fragoulidis GP, Voros D, Pafiti A. Clinicopathologic features of two rare cases of mesenchymal metastatic tumors in the pancreas: review of the literature. Pancreas 2006; 33: 301-3.

3. Oh BG, Han YH, Lee BH, Kim SY, Hwang YJ, Seo JW et al. Primary extraskeletal mesenchymal chondrosarcoma arising from the pancreas. Korean J Radiol 2007; 8: 541-4.

4. Stout AP, Verner EW. Chondrosarcoma of the extraskeletal soft tissues. Cancer 1953; 6: 581-90.

5. Casadei R, Ricci M, Ruggieri P, Biagini R, Benassi S, Picci P et al. Chondrosarcoma of the soft tissues. Two different sub-groups. J Bone Joint Surg Br 1991; 73: 162-8.

6. González-Lois C, Cuevas C, Abdullah O, Ricoy JR. Intracranial extraskeletal myxoid chondrosarcoma: case report and review of the literature. Acta Neurochir (Wien) 2002; 144: 735-40.

7. Shapeero LG, Vanel D, Couanet D, Contesso G, Ackerman LV. Extraskeletal mesenchymal chondrosarcoma. Radiology 1993; 186: 819-26.

8. Lichtenstein L, Bernstein D. Unusual benign and malignant chondroid tumors of bone. Cancer 1959; 12: 1142-57.

9. Dowling EA. Mesenchymal chondrosarcoma. J Bone Joint Surg Am 1964; 46: 747-54.

10. Nakashima Y, Unni KK, Shives TC, Swee RG, Dehlin DC. Mesenchymal chondrosarcoma of bone and soft tissue. A review of 111 cases. Cancer 1986; 57: 2444-53. 